\title{
Obstructive jaundice: Studies on predictors of biliary infection and microbiological analysis in an HIV setting
}

\author{
K S Chiliza, ${ }^{1} \mathrm{MB}$ ChB; F Madela, ${ }^{2} \mathrm{MB}$ ChB, FCS (SA), Cert Gastroenterology (SA); B Tlou, ${ }^{3}$ BSc Hons (Applied Statistics), \\ MSc (Biostatistics and Epidemiology), PhD; F Anderson, ${ }^{2} \mathrm{MB}$ ChB, FCS (SA), MMed (General Surgery), PhD \\ ${ }^{1}$ Department of Surgery, Nelson R Mandela School of Medicine, College of Health Sciences, University of KwaZulu-Natal, Durban, South Africa \\ ${ }^{2}$ Department of Surgery, Inkosi Albert Luthuli Central Hospital, Durban, South Africa \\ ${ }^{3}$ Discipline of Public Health Medicine, School of Nursing and Public Health, University of KwaZulu-Natal, Durban, South Africa
}

Correspondingauthor: K S Chiliza (kwazichiliza@yahoo.com;kwazichiliza@gmail.com)

\begin{abstract}
Background. Early diagnosis of biliary infection is critical for timely antimicrobial therapy and biliary drainage. HIV infection may influence the spectrum and severity of biliary infection in an environment with a high HIV prevalence. Charcot's triad has low sensitivity and higher specificity for biliary infection, and more sensitive markers are required.

Objectives. To investigate possible predictors of biliary infection (bacteriobilia) and identify the microbiological spectrum in patients presenting with biliary obstruction to a tertiary institute in an environment with a high prevalence of HIV.

Methods. Bile was assessed for infection at endoscopic retrograde cholangiopancreatography, percutaneous transhepatic cholangiography and surgery, and the roles of clinical/haematological factors, C-reactive protein (CRP) and procalcitonin (PCT) in determining biliary infection were evaluated.

Results: One hundred and six patients with obstructive jaundice had a mean age of 52 years (range 21 - 58); most were female (74\%), and 36 (34\%) were infected with HIV, with a mean CD4 count of 495 cells/ $\mu \mathrm{L}$. Choledocholithiasis (53\%), biliary strictures (21\%) and head of pancreas tumour (8\%) were the main aetiopathologies. Bile was obtained for microbial culture from 104 patients (98\%), and 56 (54\%) were infected. Gram-negative bacteria were most frequent (58\%), and 2 HIV-infected patients had fungal infections (Candida albicans and Aspergillus fumigatus). Screening for endoscopy-associated infections revealed Pseudomonas aeruginosa. PCT was a poor predictor of bacterial infection, whereas CRP was a fair predictor.

Conclusions. The majority of bacteria cultured were sensitive to ciprofloxacin or amoxicillin-clavulanate. Duodenoscopes were a potential source of Pseudomonas infection.
\end{abstract}

S Afr Med J 2021;111(8):803-808. https://doi.org/10.7196/SAMJ.2021.v111i8.15255

The prevalence of HIV infection in South Africa (SA) was estimated at 7.52 million cases $(13.1 \%)$ in 2018. ${ }^{[1]}$ Since HIV may affect the prevalence and severity of sepsis, it is pertinent to examine biliary sepsis in this setting of a high HIV infection rate. ${ }^{[2]}$ Bile is normally sterile, and under conditions of normal bile flow, bacteria in the biliary system are of no clinical significance. In the presence of biliary obstruction, bacteria proliferate in the stagnant bile, while biliary pressure increases. ${ }^{[3]}$ Knowledge of the spectrum of infecting organisms in a practice location facilitates early empirical antimicrobial therapy, as the infecting organism and its sensitivity may not be apparent at initial presentation. This spectrum and sensitivity may change over time. ${ }^{[4]}$ The sources of infections in the bile are reflux of duodenal contents, blood-borne infection or the portalvenous channels, and the concentration of organisms is increased in patients with cholangitis. ${ }^{[3]}$ Csendes et al. ${ }^{[5]}$ demonstrated that $>10^{5}$ organisms were seen in $0 \%$ of normal patients, $3 \%$ of patients with symptomatic gallstones, $36 \%$ of patients with common duct stones but without cholangitis, and $85 \%$ of patients with acute cholangitis. Choledocholithiasis is the leading cause of biliary obstruction, although periampullary malignancies and benign strictures have become increasingly prevalent. ${ }^{[6]}$

Acute cholangitis has a wide aetiological and clinical presentation, and may be fatal in the more severe forms. Bacterial contamination of the obstructed bile (bacteriobilia) is the initial event, which may then progress to suppurative cholangitis, liver abscesses, bacteraemia and septicaemia. ${ }^{[3]}$ The early diagnosis of acute cholangitis is critical for the initiation of antimicrobial therapy and biliary drainage. Charcot's triad is used as a marker of cholangitis, and although specific, it has been demonstrated to have sensitivity as low as $22 \%,{ }^{[5]}$ and $26.4 \%$ in a controlled study ${ }^{[6]}$ Cholangitis is currently graded as I - III based on clinical, haematological and imaging criteria. ${ }^{[7]}$

Obstructive jaundice is mainly relieved by endoscopic retrograde cholangiopancreatography (ERCP), percutaneous transhepatic cholangiography (PTC), surgical procedures and increasingly endoscopic ultrasound techniques. Non-selective relief of obstructive jaundice may result in biliary contamination and septic complications after definitive surgery for the underlying conditions. ${ }^{[3]}$ Nosocomial infection of the endoscopic equipment has also been demonstrated and linked to infections in patients. ${ }^{[8,9]}$ In patients with cholangitis, poor nutrition and renal failure, biliary decompression is necessary to manage these complications, but the benefits of preoperative biliary drainage in patients with asymptomatic bacteriobilia are unclear, as this condition is difficult to identify.

It has been noted that the spectrum of microbial agents causing acute cholangitis may change over time and result in resistant flora. Periodic evaluation is therefore required to ensure that antibiotic regimens remain relevant and effective. ${ }^{[4]}$

C-reactive protein (CRP) has been utilised for predicting biliary infection. ${ }^{[10]}$ Procalcitonin (PCT) has been proposed as of more value in predicting the outcome of sepsis than in diagnosing sepsis. ${ }^{[11]}$ 
Previous studies have not examined the aetiology and prediction of biliary infection against the background of an HIV-infected population, as is the case in SA. There are also no reliable markers of asymptomatic bacteriobilia, which requires invasive procedures for diagnosis. This lack was an additional motivation to investigate some of the known markers of sepsis in determining the presence of bacteriobilia in patients with obstructive jaundice.

\section{Objectives}

To: (i) determine the prevalence of bacteriobilia in patients presenting for therapeutic ERCP, PTC and surgery; and (ii) assess the bile microbial spectrum and antimicrobial sensitivity in patients with biliary obstruction and the association with HIV infection.

\section{Methods \\ Patients}

This was a prospective study conducted from June 2018 to June 2019. All patients aged $\geq 18$ years who presented with obstructive jaundice for ERCP, PTC or surgery were included. All patients were antibiotic naive and had not previously had ERCP, PTC or surgery. Informed consent was obtained from all patients.

\section{Methods}

At presentation, patient demographic information and HIV status (including treatment with antiretrovirals) were obtained, clinical assessment was performed (abdominal pain, temperature, pulse, blood pressure), laboratory tests for haematological, renal and liver function were carried out, and imaging by ultrasound or computed tomography was done. All patients were offered testing for HIV infection unless they were verified as infected, and those who declined testing were excluded from the study.

All patients were mentally orientated. At ERCP, PTC or surgery, 2 $5 \mathrm{~mL}$ of bile was aspirated prior to injection of contrast with singleuse biliary access devices and sterile syringes. These specimens were collected in sterile containers for microbial cultures and assessment of antimicrobial sensitivity. Antibiotic prophylaxis was not routinely used, and patients with grade II or III cholangitis were treated with ciprofloxacin empirically. The presence of biliary sepsis was defined as per the Tokyo 2018 diagnostic criteria for acute cholangitis. ${ }^{[6]} \mathrm{An}$ international normalised ratio (INR) $<1.30$ was necessary according to the unit's protocol for invasive procedures in obstructive jaundice. Specific biomarkers assessed for predicting biliary infection were clinical/haematological criteria (abdominal pain, temperature $\geq 38^{\circ} \mathrm{C}$, pulse rate $>90 \mathrm{bpm}$ prior to the procedure, white cell count $\geq 10 \times$ $10^{9} / \mathrm{L}$ ), CRP and PCT. The ERCP scope washings were also assessed for bacterial contamination and antimicrobial sensitivity on two occasions during the study.

Ethical approval was obtained from the local ethics committee of the KwaZulu-Natal Department of Health and the University of KwaZulu-Natal (ref. no. BE103/18).

\section{Statistical analysis}

All data were statistically analysed using SPSS Statistics software, version 26 (IBM, USA). Continuous variables were summarised using measures of central tendency and dispersion, while categorical variables were summarised using proportions. Using logistic regression analysis, the following independent variables were examined for their relationship with biliary infection: age, gender, ethnicity, HIV status, clinical/haematological septic markers (abdominal pain, white cell count, temperature and tachycardia), CRP and PTC. A $p$-value $<0.05$ was considered statistically significant. Receiver operating characteristic (ROC) curves were constructed and the area under the curve (AUC) was interpreted as follows: $0.60-0.69=$ poor, 0.70 $0.79=$ fair, $0.80-0.89=$ good, and $0.90-1=$ excellent.

\section{Results}

\section{Baseline clinical characteristics}

The study evaluated 106 patients during the period June 2018 - June 2019. The demographic details are set out in Table 1. The patients' ages ranged from 21 to 58 (mean 52) years, and most were female (74\%). Thirty-six patients (34\%) were infected with HIV, 65 (61\%) were not infected, and 5 (5\%) were not tested for HIV infection. The CD4 count was known in 32 patients (89\%), with a mean of 495 cells/ $\mu \mathrm{L}$ (range 19 - 1213). Choledocholithiasis (53\%), biliary strictures (21\%) and head of pancreas tumour $(8 \%)$ were the main presenting pathologies (Table 1).

\section{Biliary culture analysis and antimicrobial resistance}

Bile was obtained for culture in 104 patients (98\%), and 56 (54\%) were positive for microbes (Table 2). A single organism was found in 25 cases (45\%), and on 5 occasions (20\%) it was Pseudomonas aeruginosa. This finding was pertinent, as screening for endoscopyassociated infections only revealed $P$. aeruginosa, which was sensitive to ciprofloxacin and amoxicillin-clavulanate in 5 cases and resistant in another. Gram-negative species were most frequently cultured (58\%), with Klebsiella pneumoniae (17\%) and Escherichia coli (12\%) being the most common isolates, whereas of the Gram-positive bacteria, Streptococcus (13\%) and Enterococcus (12\%) were the most common organisms cultured. In two bile samples from HIV-infected patients, only fungal species (Candida albicans and Aspergillus fumigatus) were cultured.

Table 2 illustrates the relationship between HIV and biliary infection. Bacteriobilia was insignificantly higher in the HIVpositive patients $(p=0.86)$. The spectrum of infecting organisms was insignificantly different between HIV-positive and HIV-negative patients. The mean (standard deviation) age of the HIV-positive and HIV-negative patients was 53 (16.4) years and 48 (16.2) years, respectively $(p=0.21)$.

Of the Klebsiella and E. coli, $94 \%(n=17 / 18)$ and $92 \%(n=11 / 12)$ were sensitive to amoxicillin-clavulanate and ciprofloxacin, respectively. The other two organisms were sensitive to carbapenems. Of Pseudomonas, $86 \%(n=6 / 7)$ were sensitive to amoxicillin-clavulanate and ciprofloxacin and the remaining organism to carbapenems. All the Enterobacter, Citrobacter and Proteus species were sensitive to amoxicillin-clavulanate and ciprofloxacin. All the Streptococcus and Enterococcus species were sensitive to amoxicillin-clavulanate. Of staphylococci, $75 \%(n=3 / 4)$ were sensitive to cloxacillin, amoxicillinclavulanate and cefazolin and the remaining organism to vancomycin.

\section{Predictors of bacteriobilia}

Age and gender were not predictors of biliary infection. ROC curves were evaluated for clinical/haematological criteria, CRP and procalcitonin. The AUC for CRP was within the 'fair' range (Fig. 1).

\section{Discussion}

When compared with other studies (Table 1), the present study had more females in the cohort, but similar to the other studies, the mean age exceeded 50 years. There are inconsistencies between reported studies in the proportion of patients presenting with choledocholithiasis and malignant disease (Table 1). Choledocholithiasis is a leading cause of biliary obstruction, and this is evident in our study (53\%), but in three of the other studies malignant disease was the main cause. ${ }^{[4,12,13]}$. The $34 \%$ prevalence of HIV infection in the present study is much higher than the $16 \%$ 


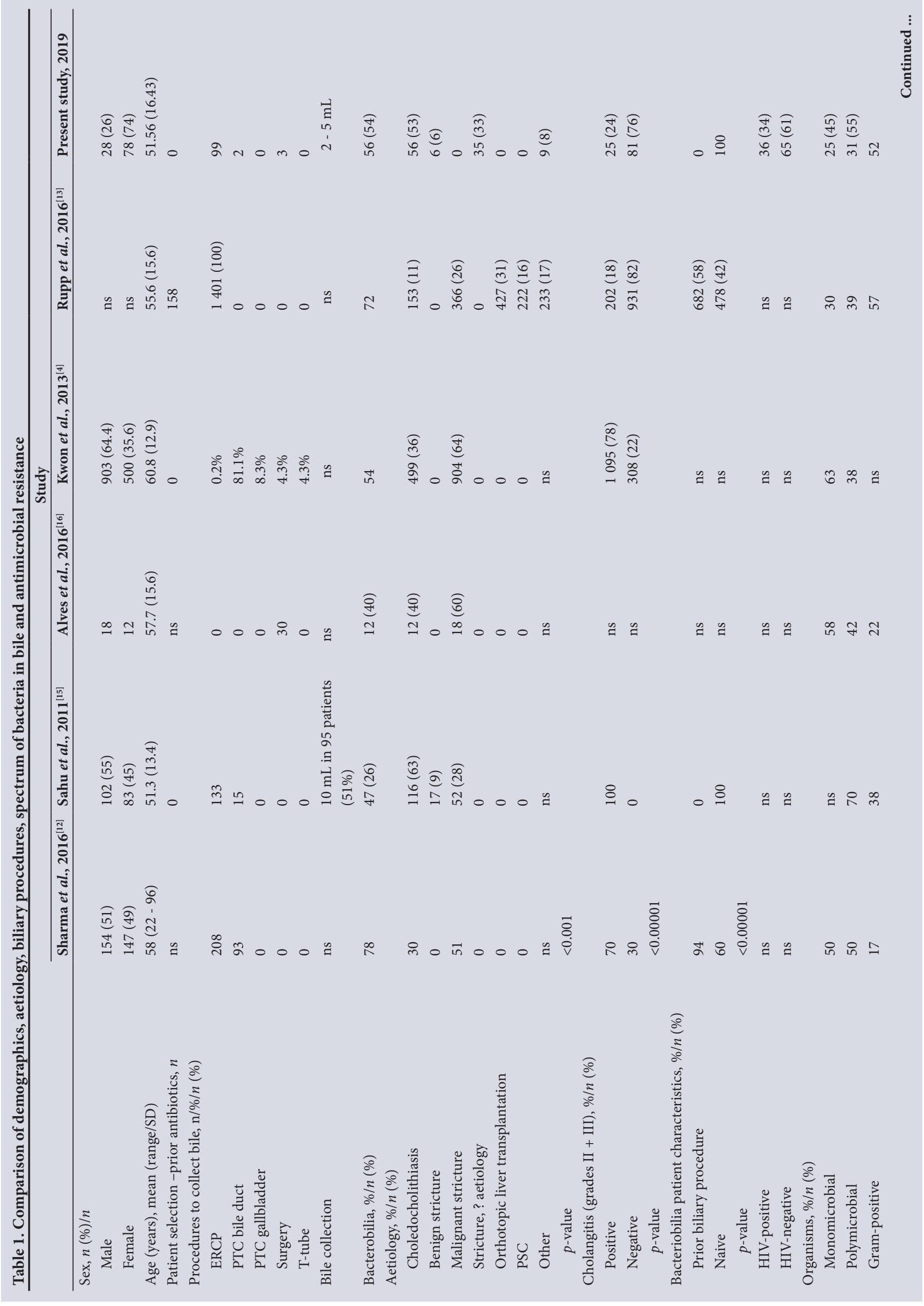




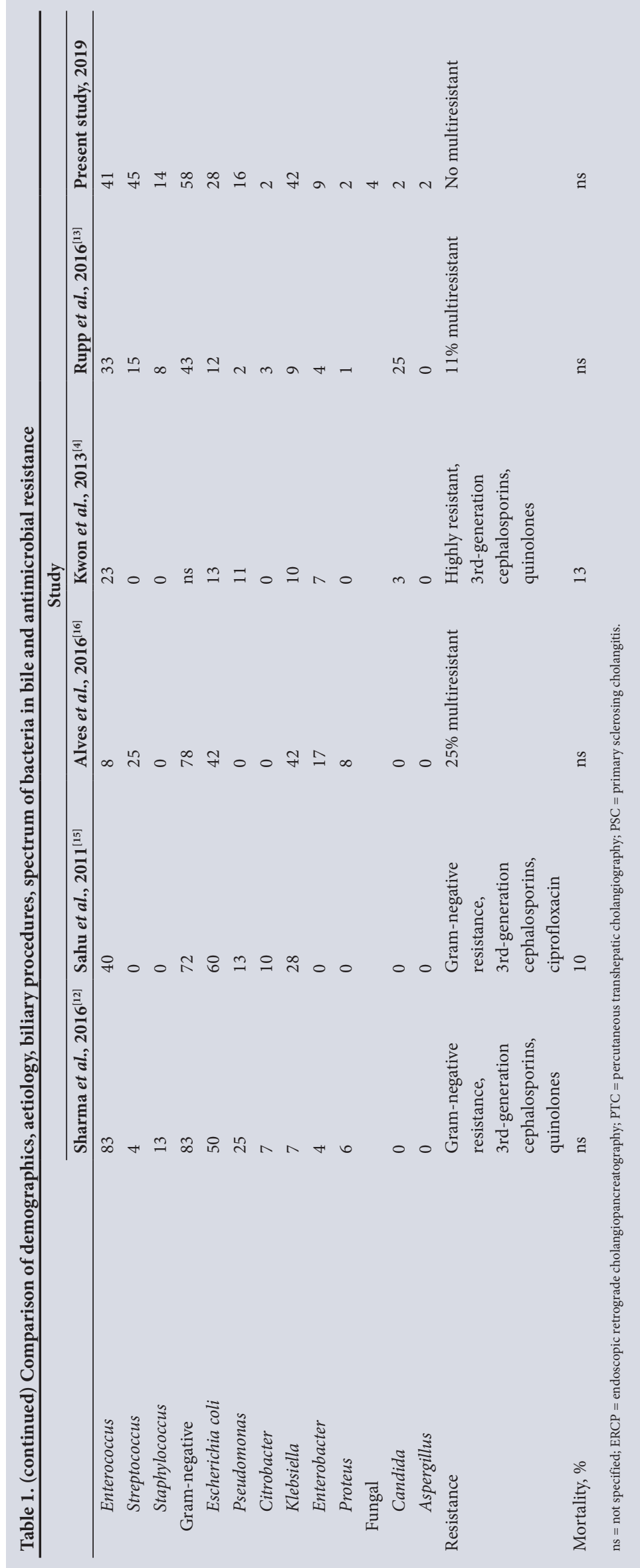

prevalence in epidemiological studies, suggesting that obstructive jaundice may be more prevalent in this group of patients, as also demonstrated by previous research. ${ }^{[14]}$

Bacteriobilia and fungobilia were identified in bile cultures of 56 patients (54\%) in the present study. The prevalence of bacteriobilia ranges from $26 \%$ to $78 \%$ in other research (Table 1).

The diagnostic and grading criteria for acute cholangitis have been revised to utilise a combination of inflammatory, cholestasis and imaging criteria to arrive at a suspected or definitive diagnosis of acute cholangitis, grading it into mild, moderate and severe forms of the complication of obstructive jaundice. ${ }^{[7]}$ Grade I (mild) cholangitis does not meet the criteria of moderate cholangitis or the presence of organ dysfunction. In grade II (moderate) cholangitis, two of the following are present: $(i)$ white cell count count $>12$ or $<4 \times 10^{9} / \mathrm{L}$; (ii) high fever $\left(\geq 39^{\circ} \mathrm{C}\right)$; (iii) age $\geq 75$ years; (iv) bilirubin $\geq 85.5 \mu \mathrm{mol} / \mathrm{L}$; and $(v)$ albumin $<24.5 \mathrm{~g} / \mathrm{L}$. Grade III (severe) disease is associated with organ dysfunction in at least one of the following systems: (i) cardiovascular: hypotension requiring dopamine $\geq 5 \mu \mathrm{g} / \mathrm{kg} / \mathrm{min}$ or any dose of norepinephrine; (ii) nervous: disturbance of consciousness; (iii) respiratory: partial pressure of oxygen/fraction of inspired oxygen ratio <300; (iv) renal: oliguria, serum creatinine $>176.84 \mu \mathrm{mol} / \mathrm{L}$; ( $v$ ) hepatic: platelets (INR $>1.5$ ); and (vi) haematological: platelet count $<100 \times 10^{9} / \mathrm{L}$. The majority of patients in the present study (76\%) had grade I cholangitis (Table 3). In three of the studies in Table 1, the majority of the patients had clinical cholangitis $(70-100 \%)$, and in one they were in the minority (18\%). In the study by Sharma et al. ${ }^{[12]}$ bacteriobilia was more frequent in patients with clinical cholangitis than in those without $(91 \%$ v. $58 \%)$. This differs from the findings of the present study, where the figures for the two groups were $40 \%$ and $54 \%$, respectively.

Although HIV was associated with a higher prevalence of infection, $58 \%(n=21 / 36)$ v. $51 \%(n=33 / 65)$ in HIV-negative patients $(p=0.8)$, the bacterial content in HIV-infected patients was similar to that in the non-HIV-infected patients, and HIV-infected patients comprised the group with fungal infections (Table 2).

Gram-negative bacilli were the dominant infecting organisms in the present study and three of the other studies (Table 1). Enterococcus was found to be the dominant organism in a Korean study ${ }^{[4]}$ and in a German study ${ }^{[13]}$ The frequent findings of streptococcal strains suggest contamination from oral flora, but these were not isolated in the endoscope washings and should be evaluated further with more frequent culture of endoscope washings.

Bacterial sensitivities varied across studies, and in the present study, the Gram-negative bacilli were sensitive to the quinolones and amoxicillinclavulanate in $86(100 \%)$ of cultures and in all but one (Staphylococcus - vancomycin) of the Grampositive bacilli. This sensitivity profile is different to that demonstrated by Sharma et al., ${ }^{[12]}$ where Gramnegative bacilli were more sensitive to carbapenems 


\section{Table 2. Spectrum of biliary microbial culture and HIV infection}

\begin{tabular}{|c|c|c|c|c|}
\hline & $\begin{array}{l}\text { HIV-positive } \\
(N=36), n(\%)\end{array}$ & $\begin{array}{l}\text { HIV-negative } \\
(N=65), n(\%)\end{array}$ & $\begin{array}{l}\text { Unknown } \\
(N=5), n(\%)\end{array}$ & $p$-value \\
\hline Biliary infection & $21(58)^{*}$ & $33(51)^{*}$ & $2(40)$ & 0.86 \\
\hline \multicolumn{5}{|l|}{ Gram-positive } \\
\hline Streptococcus species & $1(4)$ & $11(22)$ & 1 & \\
\hline Staphylococcus aureus & $2(7)$ & $2(4)$ & 0 & \\
\hline Enterococcus species & $4(14)$ & $8(16)$ & 0 & 0.18 \\
\hline \multicolumn{5}{|l|}{ Gram-negative } \\
\hline Escherichia coli & $4(14)$ & $8(16)$ & 0 & \\
\hline Klebsiella pneumoniae & $6(21)$ & $12(24)$ & 0 & \\
\hline Pseudomonas aeruginosa & $3(11)$ & $3(6)$ & 1 & \\
\hline Citrobacter freundii & $2(7)$ & $2(4)$ & 0 & \\
\hline Enterobacter cloacae & $2(7)$ & $3(6)$ & 0 & \\
\hline Proteus mirabilis & $1(4)$ & 0 & 0 & \\
\hline Morganella morganii & $1(4)$ & 0 & 0 & 0.18 \\
\hline \multicolumn{5}{|l|}{ Fungi } \\
\hline Aspergillus species & $1(4)$ & 0 & 0 & \\
\hline Candida albicans & $1(4)$ & 0 & 0 & 0.32 \\
\hline
\end{tabular}

Table 3. Grading of cholangitis in relation to bacteriobilia and PCT and CRP values

\begin{tabular}{lllll}
\hline Grade & Patients $(\boldsymbol{N = 1 0 6}), \boldsymbol{n}(\%)$ & Bacteriobilia, $\boldsymbol{n} / \mathbf{N}(\%)$ & PCT $(\mathbf{n g} / \mathbf{m L}), \mathbf{m e a n}(\mathbf{r a n g e})$ & $\mathbf{C R P}(\mathbf{m g} / \mathbf{L}), \mathbf{m e a n}(\mathbf{r a n g e})$ \\
\hline I (mild) & $81(76)$ & $44 / 81(54)$ & $2.67(0.01-17.3)$ & $56.5(1-214)$ \\
II (moderate) & $20(19)$ & $8 / 20(40)$ & $2.92(0.08-18.8)$ & $97.3(3-375)$ \\
III (severe) & $5(5)$ & $2 / 5(40)$ & $13.63(0.02-56.7)$ & $261.7(40-187)$ \\
PCT = procalcitonin; CRP = C-reactive protein. & & & &
\end{tabular}

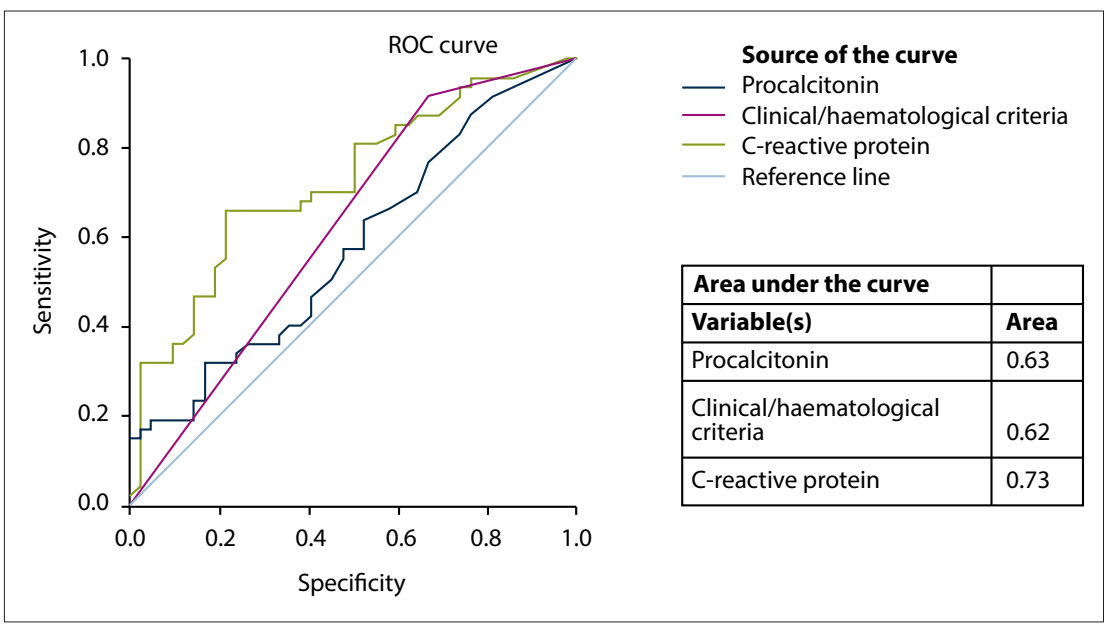

Fig. 1. ROC plots. $($ ROC $=$ receiver operating characteristic. $)$

(51 - 100\% sensitivity) and piperacillintazobactam (28-88\% sensitivity), but there was a high frequency of resistance to third-generation cephalosporins (5 $37 \%$ sensitivity) and quinolones (19 - 59\% sensitivity). These authors also demonstrated that Gram-positive cocci were less sensitive to quinolones (29-62\% sensitivity) and more sensitive to vancomycin (69-100\% sensitivity) and amoxicillin (46-76\% sensitivity). These findings are similar to those of Sahu et al., ${ }^{[15]}$ who demonstrated high resistance to the quinolones (14 $50 \%$ sensitivity) and third-generation cephalosporins (20 - 50\% sensitivity) among Gram-negative bacilli. They also found high resistance to the quinolones (26\% sensitivity) among the Gram-positive bacilli, but there was susceptibility to vancomycin (95\% sensitivity). In the German study, ${ }^{[13]}$ there was much less resistance to the quinolones, thirdgeneration cephalosporins, piperacillintazobactam and the carbapenems, with sensitivities of $75 \%, 89 \%, 87 \%$ and $99 \%$, respectively. The figures for the Grampositive bacteria were $46 \%$ and $60 \%$ for the quinolones and piperacillin-tazobactam, and $59 \%$ and $89 \%$ for the carbapenems and vancomycin.

PCT is now widely utilised in the diagnosis of bacterial infection and determining the response to antimicrobial therapy. In the present study, PCT was found to have a low sensitivity (76\%) and specificity (66\%) and an AUC of 0.58 for diagnosing bacteriobilia when all patients were assessed, which emphasises that it has value in patients who have systemic signs of infection and not localised infection. There was also no difference between the CRP and PCT values in grade I and II cholangitis, and although the values were significantly higher in grade III cholangitis, they were unable to differentiate between those with and without bacteriobilia (3.9\% v. $4.5 \%$, PCT > $10 \mathrm{ng} / \mathrm{mL}$ ).

If the present biochemical and clinical markers are poor predictors of bacteriobilia, are there other possibilities? In the study by Sahu et al., ${ }^{[15]}$ bacteriobilia was found in 12 of 30 bile samples (40\%), being more prevalent in benign disease $(n=8)$, in patients $>50$ years old $(n=1)$ and in females $(n=10) .^{[15]}$ In a study by Alves et al. ${ }^{[16]}$ of 30 bile samples, 
of which $12(40 \%)$ were infected, bacteriobilia was associated with benign biliopancreatic disease $(67 \% ; p=0.02)$, female sex $(83 \%$; $p=0.058)$ and age $>50$ years $(92 \% ; p=0.20)$, without significant associations with comorbidities $(p=0.654)$ or gallstones $(p=0.073){ }^{[16]}$ In the study by Sharma et al. ${ }^{[12]}$ of 505 bile samples, of which 396 $(78 \%)$ were infected, bacteriobilia was more frequent ( $85 \%$ v. $73 \%$; $p=0.001)$ in benign disease. In the present study, age $(p=0.208)$ and bilirubin $(p=0.38)$ had no significant influence on the presence of biliary infection.

\section{Conclusions}

Bacteriobilia in HIV-positive patients does not differ from that in the general HIV-negative population. Gram-negative bacilli remain the dominant infecting organisms, and the majority are sensitive to ciprofloxacin or amoxicillin-clavulanate and are appropriate for empirical therapy. Duodenoscopes are a potential source of Pseudomonas infection and should be periodically screened and quarantined. The majority of screened patients had grade I cholangitis.

Declaration. The research for this study was done in partial fulfilment of the requirements for KSC's MMed (Surgery) degree at the University of KwaZulu-Natal.

Acknowledgements. The authors thank the nursing team working in the gastrointestinal procedures outpatient department at Inkosi Albert Luthuli Central Hospital for their assistance in collecting blood samples. Author contributions. KSC, FA, FM: data collection and final manuscript. BT: statistics and final manuscript.

Funding. None.

Conflicts of interest. None.
1. Shisana O, Rehle T, Simbayi LC, et al. South African National HIV Prevalence, Incidence and Behaviour Survey, 2012. Cape Town: HSRC Press, 2014.

2. Huson MAM, Grobusch MP, van der Poll T. The effect of HIV infection on the host response to bacterial sepsis. Lancet Infect Dis 2015;15(1):95-108 https://doi.org/10.1016/S1473-3099(14)70917-X 3. Lipsett PA, Pitt HA. Acute cholangitis. Frontiers in Bioscience-Landmark 2003;8(6):1229-1239. https:// doi.org/10.2741/881

4. Kwon W, Jang JY, Kim EC, et al. Changing trend in bile microbiology and antibiotic susceptibilities: Over 12 years of experience. Infection 2013;41(1):93-102. https://doi.org/10.1007/s15010-012-0358-y

5. Csendes A, Diaz JC, Burdiles P, et al. Risk factors and classification of acute suppurative cholangitis. Br J Surg 1992;79(7):655-658. https://doi.org/10.1002/bjs.1800790720

6. Kiriyama S, Takada T, Strasberg SM, et al. New diagnostic criteria and severity assessment of acute cholangitis in revised Tokyo guidelines. J Hepatobiliary Pancreat Sci 2012;19(5):548-556. https://doi. cholangitis in revised Tokyo guis

7. Yokoe M, Hata J, Takada T, et al. Tokyo Guidelines 2018: Diagnostic criteria and severity grading of acute cholangitis. J Hepatobiliary Pancreat Sci 2018;25(1):17-30. https://doi.org/10.1002/jhbp.512

8. Rauwers AW, Voor in 't holt AF, Buijs JG, et al. High prevalence rate of digestive tract bacteria in duodenoscopes: A nationwide study. Gut 2018;67(9):1637-1645. https://doi.org/10.1136/ gutjinl-2017-315082

9. Ross AS, Baliga C, Verma P. A quarantine process for the resolution of duodenoscope-associated transmission of multi-drug resistant Escherichia coli. Gastrointest Endosc 2015;82(3):477-483. https:// doi.org/10.1016/j.gie.2015.04.036

10. Beliaev AM, Booth M, Rowbotham D, Bergin C. Diagnostic inflammatory markers in acute cholangitis. J Surg Res 2018;228:35-41. https://doi.org/10.1016/j.jss.2018.02.048

11. Wook Jekarl DW, Lee S, Kim M, et al. Procalcitonin as a prognostic marker for sepsis based on SEPSIS-3. Clin Lab Anal 2019;33(9):e22996. https://doi.org/10.1002/jcla.22996

12. Sharma V, Ghoshal U, Baijjal SS, et al. Frequency of biliary infection and antimicrobial susceptibility pattern in patients with extra-hepatic biliary obstruction undergoing non-surgical interventions with reused accessories. J Liver Res Disord Ther 2016;2(3):91-96. https://doi.org/10.15406/ jlrdt.2016.02.00030

13. Rupp $\mathrm{C}$, Bode $\mathrm{K}$, Weiss $\mathrm{KH}$, et al. Microbiological assessment of bile and corresponding antibiotic treatment: A strobe-compliant observational study of 1401 endoscopic retrograde cholangiographies. Medicine (Baltimore) 2016;95(10):e2390. https://doi.org/10.1097/ MDolangiographies.

14. Mbatha SZ, Anderson F. Outcomes in laparoscopic cholecystectomy in a resource constrained environment. S Afr J Surg 2016;54(3):8-12.

15. Sahu MK, Chacko A, Dutta AK, Prakash JAJ. Microbial profile and antibiotic sensitivity pattern in acute bacterial cholangitis. Indian J Gastroenterol 2011;30(5):204-208. https://doi.org/10.1007/ s12664-011-0135-3

16. Alves JR, Silva RC, Guerra SCP, et al. Microbiological analysis of bile in patients with benign and malignant biliopancreatic diseases and its consequences. Arq Gastroenterol 2016,53(3):156-162. https://doi.org/10.1007/s15010-012-0358-y

Accepted 1 March 2021 